\title{
Self-organization of Water Soluble and Amphiphile Crosslinked Carboxymethylpullulan
}

\author{
By Mélanie Legros, Virginie Dulong, Luc PICton, and Didier Le CerF*
}

The soluble state of amphiphile and crosslinked derivatives of carboxymethylpullulan was investigated by physicochemical analyses. This soluble state also called infinite sol was obtained from hydrogels after a short-time temperature treatment. The analyses show a large distribution in size for the species in solution (from some nanometers to some micrometers). Furthermore, they also reveal the presence of hydrophobic associations (intra and/or intermolecular) in addition to the chemical links. The nature of these hydrophobic interactions will depend on the polymer concentration and on the solvent used.

KEY WORDS: Polysaccharide / Crosslinking / Hydrogels / Amphiphile / Self-organization /

Hydrogels are suitable materials for drug delivery applications in biomedical and pharmaceutical fields. Indeed, active compounds can be easily entrapped and released, specially by responsive-stimuli devices which undergo phase transition or swelling-deswelling behaviour in response to environmental changes (temperature, $\mathrm{pH}$, ionic strength, ...). ${ }^{1-3}$ Furthermore, they resemble natural living tissues by their ability to absorb and retain huge quantity of fluids and by their consistency, giving them a good biocompatibility. ${ }^{4}$

Since several years, numerous studies have been interested in hydrogels based on polysaccharides and their derivatives because of their advantages over synthetic polymers. In fact, they are abundant, largely water soluble, biocompatible and descendent from renewable sources. ${ }^{5}$

More recently, attention is focused on the development of amphiphilic networks able to transport amphiphilic and hydrophobic drugs. ${ }^{6,7}$ Two majority ways are used to elaborate amphiphilic hydrogels based on polysaccharides. The most widespread way consists in grafting on the polymer backbone hydrophobic chains which self-aggregate in solution so creating cross-linking points but also hydrophobic domains. The most famous examples of this kind of systems are derivatives of pullulan bearing cholesteryl groups (CHP) which provide by self-aggregation particles able to complex hydrophobic molecules and proteins. ${ }^{8-13}$ An other example consists of carboxymethylpullulan grafting by alkyl pendant groups. ${ }^{14}$ The second technique consists in a chemical cross-linking with a hydrophobic agent. Few studies have been interested in this way although it gives in one step cross-links and amphiphilic character. So, Dulong et al. have elaborated amphiphilic carboxymethylpullulan networks using adipic dihydrazide ${ }^{15}$ and $\mathrm{N}$-hydroxysuccinimide, ${ }^{16}$ whereas Coviello and Matricardi have choosen dibromohexan as cross-linking agent to obtain hydrogels from scleroglucan ${ }^{17}$ and polygalacturonic acid. ${ }^{18}$

We use the second method and dibromohexan to elaborate amphiphilic systems based on carboxymethylpullulan, an anionic derivative of pullulan (Figure 1). In a recent paper, ${ }^{19}$ we showed that the cross-linking reaction was very fast, leading to creation of high crosslink density clusters as described by Hoffman. ${ }^{20}$ On the other hand, we demonstrated that dibromohexan gave only crosslinks and not pendant chains as we could expect. The study of the gel fraction showed that it was metastable, evolving with time and temperature to reach a soluble state called infinite sol. This transition was not induced by a change in the crosslinking ratio or in the double grafting, so, by comparing with the behaviour of associative polymers, ${ }^{21}$ we deduced that this transition was caused by an evolution of the hydrophobic interactions.

In order to understand the double structure (chemical and physical) of our systems, we will study, in this paper, thanks to physicochemical measurements, the infinite sol of hydrogels issued of low molar masses carboxymethylpullulan.

\section{MATERIALS AND METHODS}

\section{Materials}

Pullulan $\left(M_{\mathrm{n}}=120000 \mathrm{~g} \mathrm{~mol}^{-1} ; \quad M_{\mathrm{w}}=280000 \mathrm{~g} \mathrm{~mol}^{-1}\right.$ determined by SEC/MALLS measurements) was purchased from Hayashibara Biochemical Laboratory (Okayama, Japan). All reagents and solvents were commercially available and used without further purification. Water was purified with a Milli-Q reagent system (Millipore).

Synthesis and degradation of carboxymethylpullulan were described in previous works. ${ }^{19,22}$ The degree of substitution (number of carboxylic groups per anhydroglucose unit) was determined by conductimetric titration according to the method of Eyler ${ }^{23}$ and found about 0.96. The average molar masses were determined by F4/MALLS measurements (Table III).

\section{Synthesis and Chemical Characterization of Hydrogels}

Synthesis of crosslinked carboxymethylpullulan has been described in a previous work. ${ }^{19}$ Briefly, an aqueous solution of 


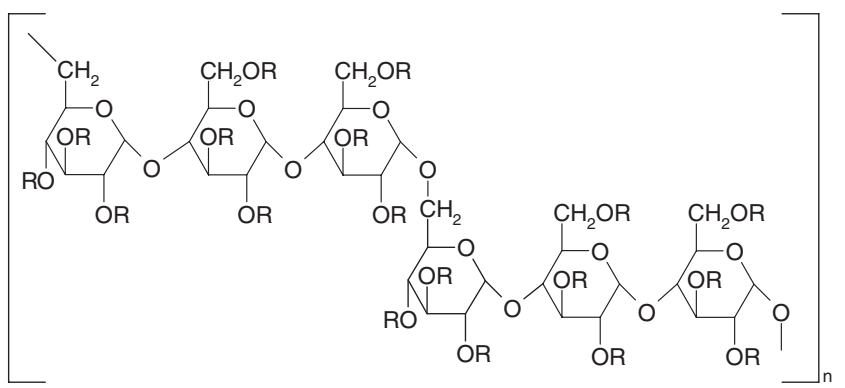

Figure 1. Structures of Pullulan $(\mathrm{R}=\mathrm{H})$ and Carboxymethylpullulan $\left(\mathrm{R}=\mathrm{CH}_{2} \mathrm{COO}^{-}, \mathrm{Na}^{+}\right)$.

$$
\mathrm{CMP}, \mathrm{N}(\mathrm{Bu})_{4} \frac{\mathrm{Br}-\left(\mathrm{CH}_{2}\right)_{6}-\mathrm{Br}}{\mathrm{DMSO}, 40^{\circ} \mathrm{C}, 2 \mathrm{~h}} \mathrm{CMP}-\left(\mathrm{CH}_{2}\right)_{6}-\mathrm{CMP}
$$

Figure 2. Synthesis of hydrogels.

$\mathrm{CMP}, \mathrm{Na}^{+}$was transformed into its acidic form $\left(\mathrm{CMP}, \mathrm{H}^{+}\right)$by elution through a cationic resin (Amberlite IRN-77, $\mathrm{H}^{+}$form) and then neutralized by tetrabutylammonium hydroxide $\left(\mathrm{Bu}_{4} \mathrm{~N}^{+}, \mathrm{OH}^{-}\right)$up to $\mathrm{pH}$ 7. After freeze-drying, the CMP, $\mathrm{Bu}_{4} \mathrm{~N}^{+}$was dissolved in dimethylsulfoxide (DMSO) at $40^{\circ} \mathrm{C}$. Dibromohexan was added under vigorous stirring and the reaction continued at the same temperature during $2 \mathrm{~h}$ with magnetic stirring (Figure 2). Hydrogels were allowed to dialyse against pure water to remove DMSO, then against $\mathrm{NaCl} 1 \mathrm{M}$ to exchange tetrabutylammonium ions to sodium ones and finally against pure water until complete removal of salts (checked by conductimetric measurements). Then the samples were filtered to separate the gel fraction of the sol fraction. Finally the sol was freeze-dried whereas the gel was precipitated in acetone and dried in a vacuum oven at $40^{\circ} \mathrm{C}$.

The incorporation rate of dibromohexan Ic was determined by gas-phase chromatography measurement of the hexanediol released after alkaline hydrolysis. ${ }^{19}$

The samples were coded as XMIc-Cp with XM: the molar mass of the CMP (LM: Low Mass, MM: Medium Mass), Ic: the incorporation rate of dibromohexan (\%) and $\mathrm{Cp}$ : the polymer concentration $\left(\mathrm{g} \mathrm{L}^{-1}\right)$. In this work, $\mathrm{Cp}$ was fixed to $200 \mathrm{~g} \mathrm{~L}^{-1}$, Ic was about $20 \%$ and only derivatives of MMCMP and LMCMP were studied, i.e., MM20-200 and LM20-200.

\section{Preparation of Solutions}

Hydrogels of MM20-200 and LM20-200 were treated during one week at $60{ }^{\circ} \mathrm{C}$ to reach the infinite sol and freezedried to recover the polymer. Then, the incorporation rate as well as the double grafting was checked by gas phase chromatography and conductimetric measurements and no changes were found for all samples.

Solutions were prepared by dissolving the polymer in the desired solvent (water, salts ...) and slowly stirred during $48 \mathrm{~h}$. Finally, samples were filtered through cellulose ester membrane (Millipore) with pore size of $8 \mu \mathrm{m}$ except in the case of surface tension and viscosity measurements.

\section{Loss of Polymer During Filtration}

The concentration of carbon in solution was measured before and after filtration with a TOC-V CSN Analyser (Shimadzu, Japan) previously calibrate with solutions of potassium hydrogen phthalate. The percentage of polymer lost during filtration was calculated according to eq 1 where $(C)_{b f}$ is the carbon concentration before filtration and $(\mathrm{C})_{\mathrm{af}}$ the concentration after filtration with a relative uncertainty of $5 \%$ :

$$
\text { Loss }=\frac{(\mathrm{C})_{\mathrm{bf}}-(\mathrm{C})_{\mathrm{af}}}{(\mathrm{C})_{\mathrm{bf}}} \times 100
$$

\section{Quasi-Elastic Light Scattering (QELS) Measurements}

QELS measurements were performed at $25^{\circ} \mathrm{C}$ for a polymer concentration of $1 \mathrm{~g} \mathrm{~L}^{-1}$ using a Zêtasizer NanoZS equipped with a He-Ne laser (632 nm) (Malvern Instruments Ltd, U. K.). Results were analysed using the DTS Dispersion Technology software (v. 4.00, Malvern Instruments, United Kingdom) by a cumulant method and the hydrodynamic diameter is obtained from an average of ten measurements with a mean relative uncertainty of $10 \%$.

\section{Coupling Flow-field Flow Fractionation-multi-angle Laser Light Scattering (F4/MALLS) Measurements}

Determination of molar masses was done with a flow-field flow fractionation (F4) coupled online with a multi-angle laser light scattering (MALLS) and a differential refractive index detector (DRI). ${ }^{24-26}$

The F4 is a universal fractionator model F-1000 from Postnova analytics (Germany). The linear channel flow rate $\left(\mathrm{F}_{\mathrm{L}}\right)$ is regulated with an intelligent pump HPLC flom 301 (FLOM, Japan), while the crossflow $\left(\mathrm{F}_{\mathrm{C}}\right)$ is generated by a $\mathrm{P}$ 500 dual piston syringe pump (Pharmacia, France) piloted by the FF Universal Fractionators Flow software (v. 1.60). The sample-injected volume consists of a $100 \mu \mathrm{L}$ full loop. $\mathrm{LiNO}_{3}$ or $\mathrm{KSCN} 0.1 \mathrm{M}$ used as carrier was filtered through $0.1 \mu \mathrm{m}$ filter unit (Millipore) and degassed (ERC-413). For all measurements, we have respected the following conditions: $\mathrm{F}_{\mathrm{L}}=$ $0.5 \mathrm{~mL} \mathrm{~min}^{-1}, \mathrm{~F}_{\mathrm{C}}=0.8 \mathrm{~mL} \mathrm{~min}^{-1}$ for $5 \mathrm{~min}$, a decay of $5 \mathrm{~min}$ (exponential factor -1) until $0.3 \mathrm{~mL} \mathrm{~min}^{-1}$, a plateau at 0.3 $\mathrm{mL} \min ^{-1}$ for $5 \mathrm{~min}$ then a last plateau at $0.05 \mathrm{~mL} \mathrm{~min}^{-1}$ until the end of analysis.

F4 is coupled on line with MALLS and DRI. The MALLS photometer, an EOS from Wyatt Technology inc. (USA) is equipped with a K5 cell and a GaAs laser $(690 \mathrm{~nm})$. The concentration of each eluted fraction has been determined with the DRI (ERC 7515A) from the measured value of $\mathrm{dn} / \mathrm{dC}$ $\left(0.150 \mathrm{~mL} \mathrm{~g}^{-1}\right.$ for all the samples). The collected data were analysed using the Astra v. 4.90 software package (Wyatt Technology inc., USA).

The polymer concentration was fixed at $5 \mathrm{gL}^{-1}$ for the LM20-200 and $2 \mathrm{~g} \mathrm{~L}^{-1}$ for the MM20-200.

\section{Surface Tension Measurements}

Surface tension was measured at $25^{\circ} \mathrm{C}$ using the Wilhelmy plate method with a K-12 tensiometer (Krüss, Germany) and a 


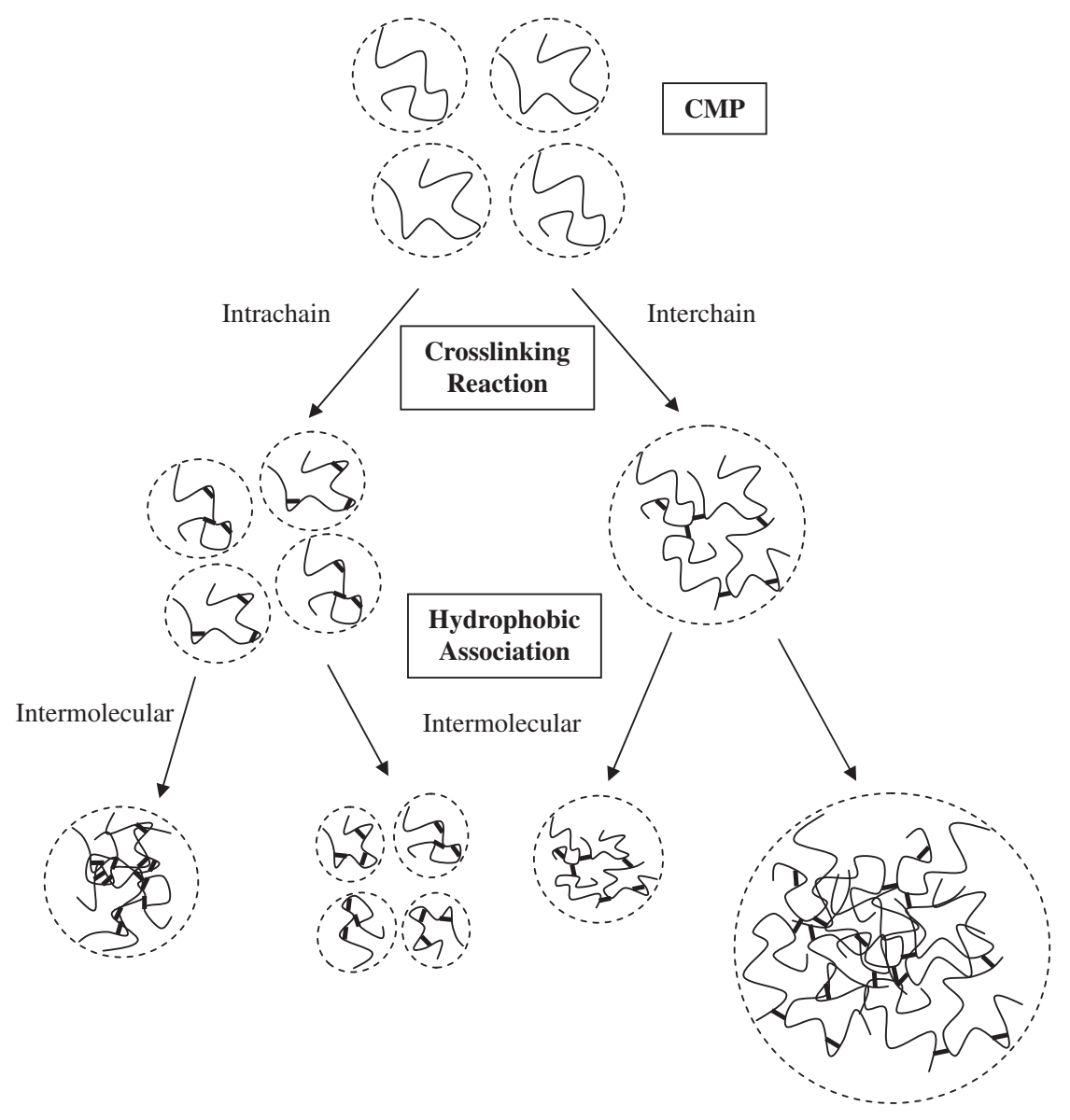

Figure 3. Schematic representation of the various possible structures of species in solution.

platinum plate. Data were analysed using the K122 software (Krüss, Germany). Measurements were performed at a polymer concentration of $1 \mathrm{~g} \mathrm{~L}^{-1}$ for an equilibration time of $3600 \mathrm{~s}$. Each equilibrium surface tension was the mean of three measurements with an uncertainty of $0.2 \%$.

\section{Viscosity Measurements}

Viscosity measurements were performed at $25^{\circ} \mathrm{C}$ in the Newtonian domain at low shear rate $\left(\sim 5 \mathrm{~s}^{-1}\right)$ using a Contraves LS30 viscosimeter. Specific viscosity $\left(\eta_{\text {spe }}\right)$ was calculated according to eq 2 with $\eta$ : the viscosity of the sample and $\eta_{0}$ : the viscosity of the solvent:

$$
\eta_{\text {spe }}=\frac{\eta-\eta_{0}}{\eta_{0}}
$$

\section{RESULTS AND DISCUSSION}

Before analysing the results of the physicochemical study, we will sum up, to ameliorate the understanding of the observed phenomena, the various possible structures of the species in solution according to the nature of chemical links and physical interactions (Figure 3).

On one hand, chemical crosslinks can form between several polymer chains (interchain) and/or within the same chain (intrachain) giving what we called "crosslinked objects." On the other hand, hydrophobic interactions can be added to chemical links between (intermolecular) and/or within (intramolecular) the crosslinked objects. This double organization gives birth to numerous possibilities of structures and so to a great variety of species in solution. Furthermore, we can also expect evolutions of hydrophobic interactions (transition between intramolecular and intermolecular associations) depending on the polymer concentration and on the environment as it was observed with hydrophobic derivatives of CMP. ${ }^{27}$

This means that the study of our systems will be particularly complex because of the diversity of their structures but also because of their ability to evolve.

\section{Effect of Filtration}

Filtration can affect the results of the physicochemical analyses if a great part of the sample is retained on the membrane. So, we decided to determine the loss of polymer during filtration through a membrane with pore size of $8 \mu \mathrm{m}$. This will also give information about the size distribution of the species in solution. The results for MM20-200 and LM20-200 in water and in $\mathrm{LiNO}_{3} 0.1 \mathrm{M}$ are summarized in Table I.

First, we observe that the percentage of loss increases hugely with the molar mass of the precursor whatever the solvent. It means that the structures in MM20-200 are larger 
Table I. Loss of polymer during filtration (\%)

\begin{tabular}{ccc}
\hline Samples & Water & $\mathrm{LiNO}_{3} 0.1 \mathrm{M}$ \\
\hline MM20-200 & 41 & 59 \\
LM20-200 & 9 & 4 \\
\hline
\end{tabular}

than those in LM20-200. It can be explained by the level of entanglement during the synthesis of hydrogels. In the case of MM20-200, it is higher because of the higher molar mass and it increases the probability to hang several polymer chains together and so to obtain larger species.

The losses are also dependant on the solvent. The use of $\mathrm{LiNO}_{3}$ decreases slightly the retention for LM20-200 but increases of about $20 \%$ the losses for MM20-200. The decrease of electrostatic repulsions can induce either more compact structures as observed for LM20-200, or an increase in size by favouring intermolecular hydrophobic interactions as it seems to be the case for MM20-200.

From these results, we can conclude that the losses are negligible for LM20-200 but not for MM20-200. Its characterization will be affected by an important retention on the membrane and give only a partial view of the species in solution.

\section{Determination and Evolution of Hydrodynamic Diameters}

We have studied the evolution of the hydrodynamic diameters of MM20-200 and LM20-200 in water, in a lyotropic salt $(\mathrm{NaCl})$ and in a chaotropic salt (KSCN) (Table II). We have chosen two types of salts in order to estimate the impact of hydrophobic interactions in the structures of our systems. Indeed, $\mathrm{NaCl}$ will strengthen these interactions while $\mathrm{KSCN}$ will decrease them. ${ }^{28}$ Hydrodynamic diameters of precursors are also indicated for comparison.

For MM20-200, we have to remind that $60 \%$ of the sample is analysed in water and only $40 \%$ in salt solutions. This means that a great part, even a major part, of the species is very large either by crosslinking of numerous polymer chains and/or by intermolecular hydrophobic associations. So, the hydrodynamic diameters given in Table II correspond to the smaller species. In water, we observe that the Dh of MM20-200 is slightly smaller than that of its precursor. It can be the consequence of the crosslinking reaction between several polymer chains or inside a same chain which will give more compact structures. However, we can conclude to the absence of intermolecular hydrophobic associations certainly prevented by electrostatic repulsions. In $\mathrm{NaCl} 0.1 \mathrm{M}$, we observe a decrease of the hydrodynamic diameter due to the screening of

Table II. Evolution of hydrodynamic diameters $(\mathrm{nm})$ of the precursors (MMCMP and LMCMP) and of the infinite sol of MM20-200 and LM20-200 in water, $\mathrm{NaCl} 0.1 \mathrm{M}$ and $\mathrm{KSCN} 0.1 \mathrm{M}$

\begin{tabular}{cccc}
\hline Samples & Water & $\mathrm{NaCl} 0.1 \mathrm{M}$ & KSCN 0.1 M \\
\hline MMCMP & 79 & 7.5 & 7.5 \\
MM20-200 & 68 & 44 & 10 \\
LMCMP & 59 & 4 & 4 \\
LM20-200 & 51 & 7.5 & 7.5 \\
\hline
\end{tabular}

the charges but also to the lyotropic character of $\mathrm{NaCl}$, which will strengthen, in this case, the hydrophobic interactions. In $\mathrm{KSCN}$, the hydrodynamic diameter is smaller than in $\mathrm{NaCl}$. The ionic strength being constant, we can explain this decrease by the dissociation of intermolecular hydrophobic associations between the crosslinked objects. However, we could not determine the losses in polymer because of the presence of carbon in this salt, so we can not assert that this observation concerns the majority of the sample.

Concerning LM20-200, we are sure that the results obtained are representative of the entire sample, because of the very weak retention during filtration. As for MM20-200, the hydrodynamic diameter of LM20-200 in water is smaller than its precursor. However, in both salts, we obtain the same value of hydrodynamic diameter. Two hypotheses can explain this observation. First, it is possible that, in $\mathrm{KSCN}$, the screening effect is predominant over the dissociation. Secondly, this sample can possess few hydrophobic interactions and the small $\mathrm{Dh}$ is due only to the screening of the electrostatic repulsions. The obtained results do not permit us to validate one of the hypotheses.

\section{F4/MALLS Measurements}

For these measurements, we have worked with more concentrated solutions $\left(5 \mathrm{~g} \mathrm{~L}^{-1}\right.$ for LM20-200 and $2 \mathrm{~g} \mathrm{~L}^{-1}$ for MM20-200) in order to obtain a sufficient response in LS. It means that no comparison between QELS and F4/MALLS/ DRI measurements will be possible.

Figures 4 and 5 illustrated the fractionation profiles (both DRI and LS) together with the molar masses distributions of LMCMP and LM20-200 in $\mathrm{LiNO}_{3}$ 0.1 M and in KSCN 0.1 M.

Fractograms for MMCMP and MM20-200 are not shown, but Table III summarizes their results and those of LMCMP and LM20-200. (i.e., the main DRI responses).

For LM20-200 and MM20-200 in both solvents, two populations are evidenced by MALLS detector, whereas only one population is observed according DRI response (proportional to the concentration). The second population is eluted at higher elution volume (superior to $8 \mathrm{~mL}$ ) and shows an important LS signal with little DRI response. It evidences the presence of very large structures in size but in very low proportion. The first population represents almost all the detected mass (DRI response) and its characteristics are reported in Table III. Concerning the precursors (MMCMP

Table III. Characteristics of precursors (MMCMP and LMCMP) and crosslinked systems (MM20-200 and LM20-200) according to the DRI response from F4/MALLS measurements in $\mathrm{LiNO}_{3} 0.1 \mathrm{M}$ and $\mathrm{KSCN} 0.1 \mathrm{M}$

\begin{tabular}{lcccccc}
\hline & \multicolumn{3}{c}{$\mathrm{LiNO}_{3} 0.1 \mathrm{M}$} & \multicolumn{3}{c}{$\mathrm{KSCN} \mathrm{0.1 \textrm {M }}$} \\
\hline Samples & $\begin{array}{c}M_{\mathrm{n}} \\
\left(\mathrm{g} \mathrm{mol}^{-1}\right)\end{array}$ & $\begin{array}{c}M_{\mathrm{w}} \\
\left(\mathrm{g} \mathrm{mol}^{-1}\right)\end{array}$ & $\mathrm{Rg} / \mathrm{M}$ & $\begin{array}{c}M_{\mathrm{n}} \\
\left(\mathrm{g} \mathrm{mol}^{-1}\right)\end{array}$ & $\begin{array}{c}M_{\mathrm{w}} \\
\left(\mathrm{g} \mathrm{mol}^{-1}\right)\end{array}$ & $\mathrm{Rg} / \mathrm{M}$ \\
\hline MMCMP & 61000 & 115000 & $/$ & $/$ & $/$ & $/$ \\
\hline MM20-200 & 57000 & 144000 & 0.3 & 43000 & 70000 & 0.5 \\
\hline LMCMP & 30000 & 40000 & $/$ & $/$ & $/$ & $/$ \\
\hline LM20-200 & 80000 & 380000 & 0.3 & 36000 & 54000 & 0.5
\end{tabular}




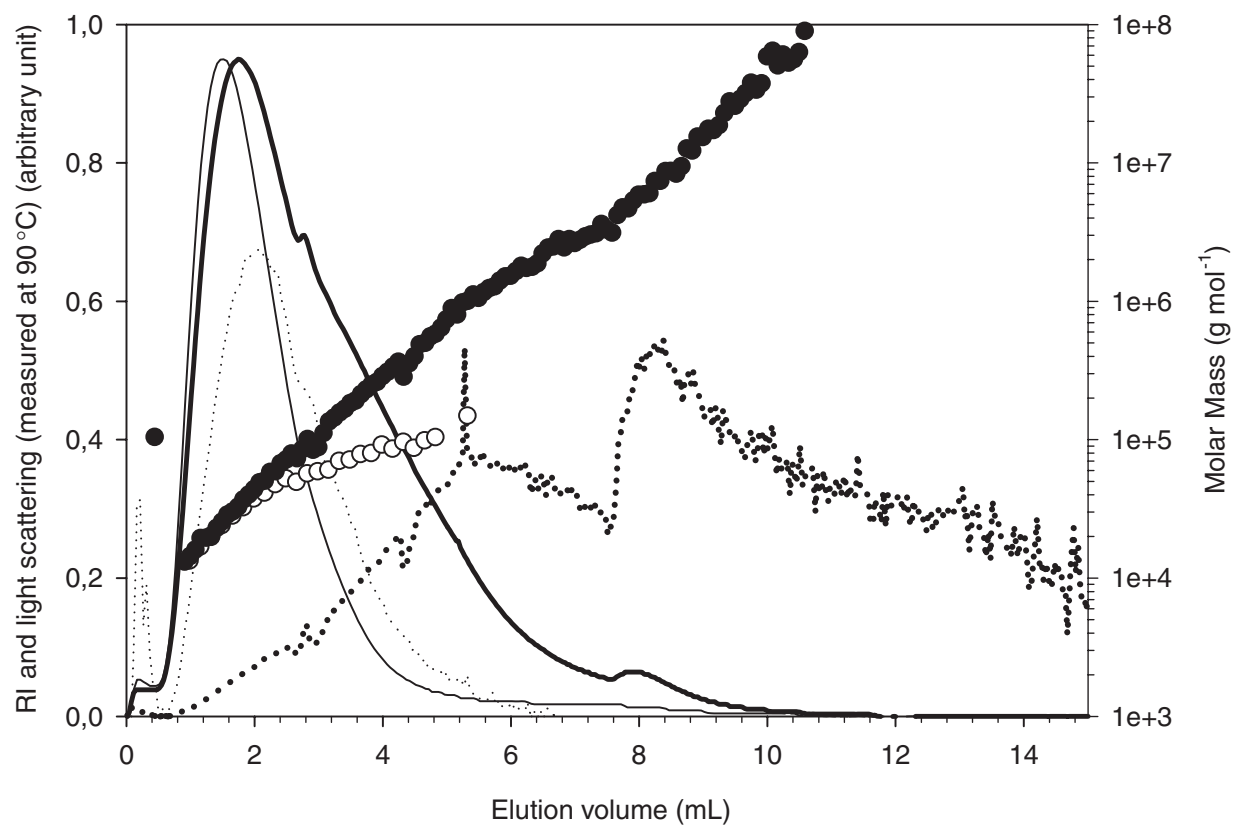

Figure 4. Elution profiles from refractive index (full lines) and light scattering (dotted lines) of LMCMP (hairline) and LM20-200 (bold line) together with molar mass distributions of LMCMP $(\bigcirc)$ and LM20-200 (O) determined by F4/MALLS in LiNO $30.1 \mathrm{M}, \mathrm{C}=5 \mathrm{~g} \mathrm{~L}^{-1}$.

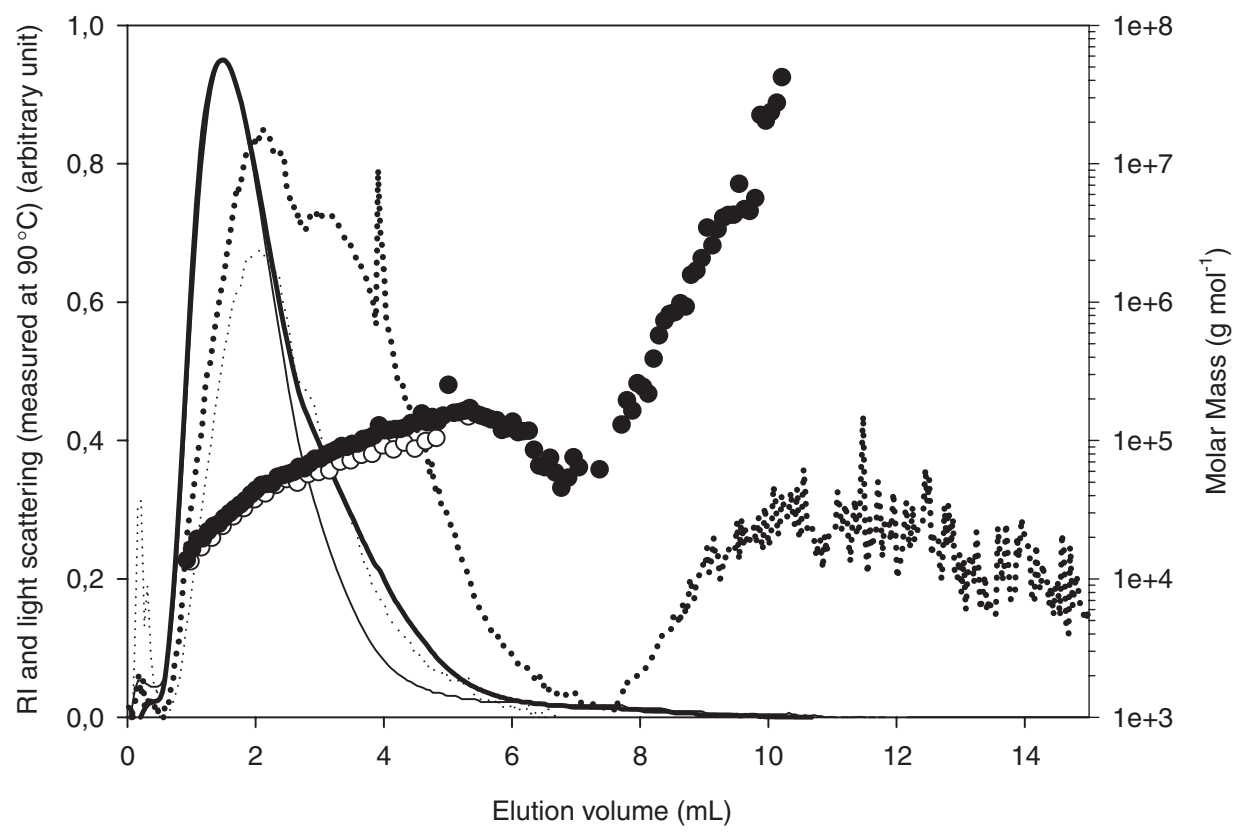

Figure 5. Elution profiles from refractive index (full lines) and light scattering (dotted lines) of LMCMP (hairline) and LM20-200 (bold line) together with molar mass distributions of LMCMP $(\bigcirc)$ and LM20-200 $(\bullet)$ determined by F4/MALLS in KSCN $0.1 \mathrm{M}, \mathrm{C}=5 \mathrm{~g} \mathrm{~L}^{-1}$.

and LMCMP), only one population is detected and results are independent of the solvent.

In Figure 4, we can observe that the molar masses distribution of LM20-200 in $\mathrm{LiNO}_{3}$ deviates from those of the LMCMP, what is visible in the values of $M_{\mathrm{n}}$ and $M_{\mathrm{w}}$.

However, in $\mathrm{KSCN}$, its distribution is almost superimposed on those of the precursor, with values of $M_{\mathrm{n}}$ and $M_{\mathrm{w}}$ quite identical. This difference between the two salts indicates clearly the presence of intermolecular associations. These association phenomena are probably caused by the increase of the concentration in comparison with those used for QELS measurements. It means also that we do not observe here isolated objects and we can not conclude on the nature of the crosslinks in this sample.

Concerning MM20-200, we observe in $\mathrm{LiNO}_{3}$ no difference in $M_{\mathrm{n}}$ values between the sample and its precursor, whereas $M_{\mathrm{w}}$ is slightly higher. This indicates the existence of intermolecular associations confirmed by the results in KSCN. However, in 
this solvent, the values of molar masses (both $M_{\mathrm{n}}$ and $M_{\mathrm{w}}$ ) are smaller than those of the MMCMP. Such behaviour in the case of hydrophobically modified $\mathrm{CMP}^{27}$ was explained by the presence of strong intramolecular associations inducing a compactness of the macromolecule. For our sample, which is also crosslinked, it can be explain both by intrachain crosslinking and/or intramolecular interactions. However, we have observed previously that MM20-200 is more or less retained during filtration. So it is possible that the observed structures are not majority in the sample.

More precise conformational information of these samples can be obtained from examination of the molar mass dependence of the size parameters (gyration radii), which is well described by power law. For non crosslinked samples the gyration radii are too small to be obtained by MALLS (lower than $20 \mathrm{~nm}$ ). The calculated values of the various exponents are listed in Table II for the higher species (higher volume of the first population). The reported data show that the two samples in $\mathrm{LiNO}_{3}$ are characterized by lower values of power-law exponents. It suggests a more compact conformation owing to intramolecular interactions. The higher power-law exponents in $\mathrm{KSCN}$ show a less compact conformation by destruction of intramolecular interactions in addition to the destruction of intermolecular interaction (decrease of $M_{\mathrm{w}}$ ) by chaotropic salt.

\section{Surface Tension Measurements}

For this study, in order to observe the overall sample, we have decided not to filter them. We have worked for both systems at a concentration of $1 \mathrm{~g} \mathrm{~L}^{-1}$. The values of equilibrium surface tension in water, $\mathrm{NaCl} 0.1 \mathrm{M}$ and $\mathrm{KSCN} 0.1 \mathrm{M}$ are summarized in Table IV.

First, we observe that these values are similar whatever the sample and the solvent. Furthermore, they are close of the surface tension of pure water $\left(\sim 72 \mathrm{mN} \mathrm{m}^{-1}\right)$. It indicates that our systems do not adsorb at the air/water interface. Such results have been obtained for amphiphilic polysaccharides, in particular hydrophobically modified alginate ${ }^{29}$ or pectin ${ }^{30}$ with dodecyl groups and cholesteryl bearing pullulan. ${ }^{8}$ These systems form aggregates in solution with the hydrophobic parts inside and the hydrophilic areas exposed to water. This conformation is caused either by the stiffness of the structures or by the important quantity or length of hydrophobic groups. In our case, our systems are also crosslinked. It induces on one hand stiffer structures and on the other hand less mobile hydrophobic areas.

\section{Viscosity Measurements}

We have not filtered our samples before measurements to avoid losses of polymer. Furthermore, we have worked with more concentrated solutions (superior to $10 \mathrm{~g} \mathrm{~L}^{-1}$ ). It induces

Table IV. Equilibrium surface tensions $\left(\mathrm{mN} \mathrm{m}^{-1}\right)$ of MM20-200 and LM20-200 in water, $\mathrm{NaCl} 0.1 \mathrm{M}$ and $\mathrm{KSCN} 0.1 \mathrm{M}, \mathrm{C}=1 \mathrm{~g} \mathrm{~L}^{-1}$

\begin{tabular}{cccc}
\hline Samples & Water & $\mathrm{NaCl} 0.1 \mathrm{M}$ & KSCN 0.1 M \\
\hline MM20-200 & 65 & 71 & 66 \\
LM20-200 & 68 & 68 & 68 \\
\hline
\end{tabular}

that the obtained structures will not be isolated and that we will observe association phenomena.

Figures 6 and 7 illustrated the evolution of specific viscosity of MM20-200 and LM20-200 versus concentration in water, in $\mathrm{NaCl} 0.1 \mathrm{M}$ and in $\mathrm{KSCN} 0.1 \mathrm{M}$. The systems have a great tendency to self-associate. Consequently, we have not determined the intrinsic viscosity, which is characteristic of an isolated macromolecule.

We observe that the specific viscosity in water for MM20200 is always higher, in the range of studied concentration, than those in salts. This behaviour is due to electrostatic repulsions which are more important in water than hydrophobic interactions. The nature of the salt does not change the evolution and the values of specific viscosity. Two hypotheses can explain this behaviour. First, it is possible that the screening effect of the salts prevails and induces reinforcements of hydrophobic associations. Then, the formed clusters can be too strong to be dissociated by KSCN. Secondly, this behaviour could be due to the absence of hydrophobic associations. However, this second hypothesis is slightly probable in comparison with the observations made in the previous analyses, which show the presence of associations at lower concentrations.

For LM20-200, we observe also specific viscosities higher than those in salts because of electrostatic repulsions. In $\mathrm{NaCl}$ $0.1 \mathrm{M}$, a decrease of specific viscosities is noted due to screening of charges but also to reinforcements of intramolecular interactions. The use of $\mathrm{KSCN}$ induces an increase of the values of specific viscosity comparing to $\mathrm{NaCl}$. This phenomenon is caused by the dissociation of intramolecular associations leading to an increase of the hydrodynamic volume of the species in solution according to F4/MALLS/ DRI.

\section{CONCLUSION}

The physicochemical characterization of the infinite sol of MM20-200 and LM20-200 has showed the presence of hydrophobic interactions (intra and/or intermolecular). It confirms the double organization (chemical and physical) of our systems, already observed in the gel fraction. This study has also highlighted the high complexity of the species in solution. Indeed, they vary by their intrinsic structure and by their size (from some nanometers to some micrometers). Furthermore, they are dependant on the concentration, the solvent and the conditions used for the analyses (filtration, concentration ...). However, these new systems, which behave like associative derivatives, can have an interesting potential as carrier material thanks to their ability to associations but also to evolve in function of their environment.

Acknowledgment. We would to thank the "French Ministère de l'Education Nationale, de la Recherche et de la Technologie $\gg$ for financial support. We would also thank the "réseau Matériaux, Polymères, Plasturgie (MPP)" and the "région Haute-Normandie" for financial help. 


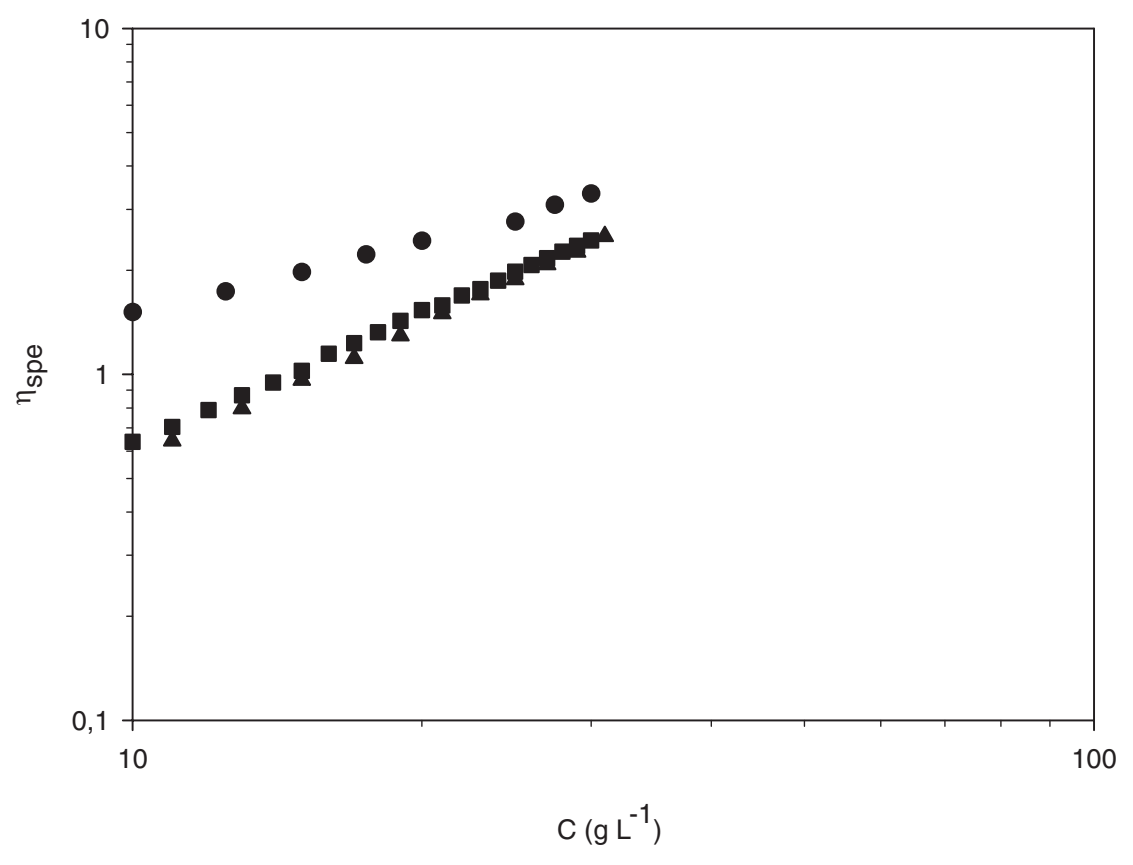

Figure 6. Evolution of $\eta_{\text {spe }} v s . \mathrm{C}$ for MM20-200 in water (O), $\mathrm{NaCl} 0.1 \mathrm{M}(\boldsymbol{\square})$ and $\mathrm{KSCN} 0.1 \mathrm{M}(\boldsymbol{\Delta})$.

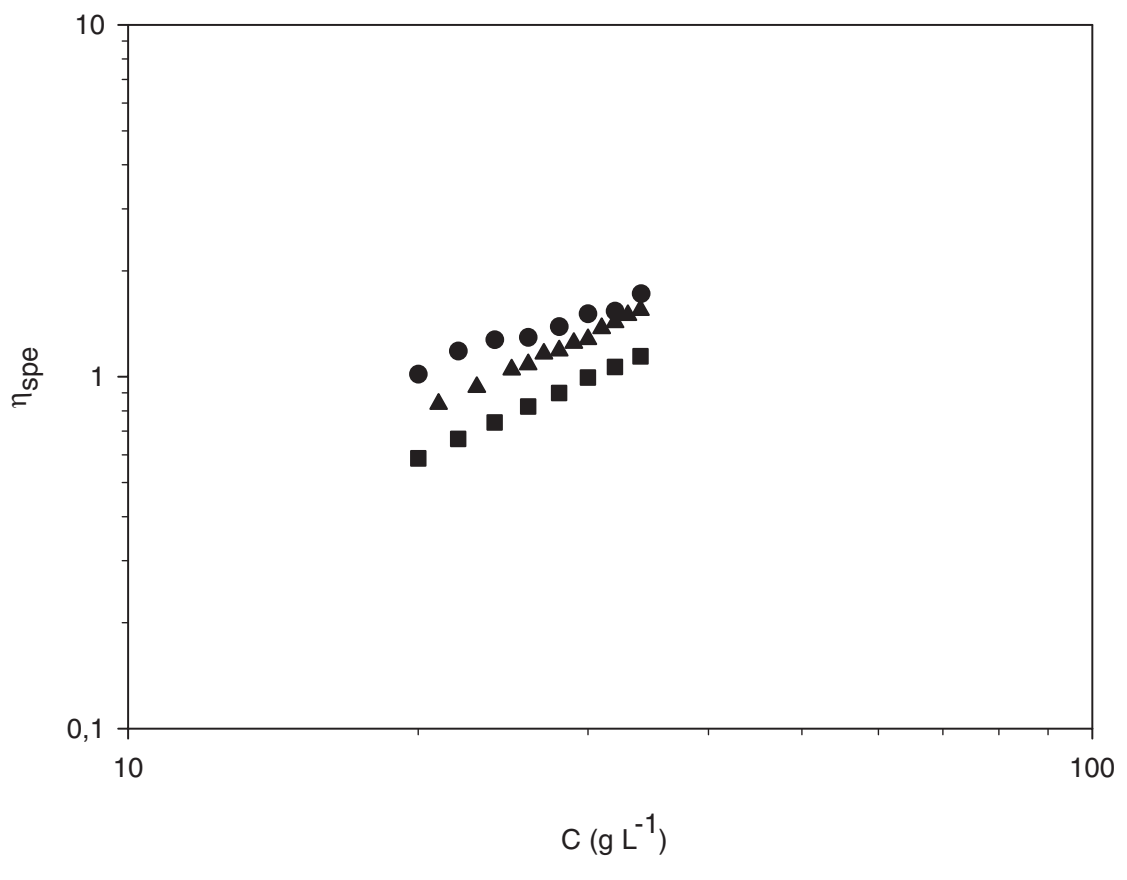

Figure 7. Evolution of $\eta_{\text {spe }}$ vs. C for LM20-200 in water (O), $\mathrm{NaCl} 0.1 \mathrm{M}(\boldsymbol{\square})$ and $\mathrm{KSCN} 0.1 \mathrm{M}(\boldsymbol{\Delta})$.

Received: May 13, 2008 Accepted: August 12, 2008 Published: October 8, 2008

\section{REFERENCES}

1. N. A. Peppas, P. Bures, W. Leobandung, and H. Ichikawa, Eur. J. Pharm. Biopharm., 50, 27 (2000).

2. Y. Qiu and K. Park, Adv. Drug Delivery Rev., 53, 321 (2001).
3. S. Chaterji, I. K. Kwon, and K. Park, Prog. Polym. Sci., 32, 1083 (2007).

4. "Hydrogels for Medical and Biomedical Applications," B. D. Ratner, A. S. Hoffman, J. D. Andrade Ed., ACS Symposium series, $\mathrm{N}^{\circ} 31$ American Chemical Society, Washington, DC, 1976, p. 1.

5. T. Coviello, P. Matricardi, C. Marianecci, and F. Alhaique, J. Controlled Release, 119, 5 (2007).

6. C. S. Patrickios and T. K. Georgiou, Curr. Opin. Colloid In., 8, 76 (2003).

7. T. R. Hoare and D. S. Kohane, Polymer, 49, 8 (2008). 
8. K. Akiyoshi, S. Deguchi, N. Moriguchi, S. Yamaguchi, and J. Sunamoto, Macromolecules, 26, 3062 (1993).

9. K. Akiyoshi, T. Nishikawa, Y. Mitsui, T. Miyata, M. Kodama, and J. Sunamoto, Colloid Surf., A, 112, 91 (1996).

10. K. Akiyoshi, S. Kobayashi, S. Shichibe, D. Mix, M. Baudys, S. W. Kim, and J. Sunamoto, J. Controlled Release, 54, 313 (1998).

11. K. Kuroda, K. Fujimoto, J. Sunamoto, and K. Akiyoshi, Langmuir, 18, 3780 (2002).

12. T. Nishikawa, K. Akiyoshi, and J. Sunamoto, J. Am. Chem. Soc., 118, 6110 (1996).

13. T. Nishikawa, K. Akiyoshi, and J. Sunamoto, Macromolecules, 27, 7654 (1994).

14. W. Henni-Silhadi, M. Deyme, M. M. Boissonnade, M. Appel, D. Le Cerf, L. Picton, and V. Rosilio, Pharm. Res., 24, 2317 (2007).

15. V. Dulong, D. Le Cerf, L. Picton, and G. Muller, Colloids Surf. A, 274, 163 (2006).

16. V. Dulong, G. Mocanu, and D. Le Cerf, Colloid Polym. Sci., 285, 1085 (2007).

17. T. Coviello, M. Grassi, G. Rambone, E. Santucci, M. Carafa, E. Murtas, F. M. Riccieri, and F. Alhaique, J. Controlled Release, 60, 367 (1999).

18. P. Matricardi, M. Dentini, V. Crescenzi, and S. B. Ross-Murphy,
Carbohydr. Polym., 27, 215 (1995).

19. M. Legros, P. Cardinael, V. Dulong, L. Picton, and D. Le Cerf, Polym. J., 40, 233 (2008).

20. A. S. Hoffman, Adv. Drug Delivery Rev., 43, 3 (2002).

21. S. Simon, G. Mocanu, L. Picton, D. Le Cerf, and G. Muller, e-polymers, 38, 1 (2004).

22. I. Bataille, J. Huguet, G. Muller, G. Mocanu, and A. Carpov, Int. J. Biol. Macromol., 20, 179 (1997).

23. R. W. Eyler, T. S. Klug, and F. Siephuis, Anal. Chem., 19, 24 (1947).

24. L. Picton, I. Bataille, and G. Muller, Carbohydr. Polym., 42, 23 (2000).

25. D. Le Cerf, S. Simon, J. F. Argillier, and L. Picton, Anal. Chim. Acta, 604, 2 (2007).

26. C. Duval, D. Le Cerf, L. Picton, and G. Muller, J. Chromatogr., B, 753, 115 (2001).

27. S. Simon, J. Y. Dugast, D. Le Cerf, L. Picton, and G. Muller, Polymer, 44, 7917 (2003).

28. L. Picton and G. Muller, Prog. Colloid Polym. Sci., 102, 26 (1996).

29. V. G. Babak, E. A. Skotnikova, I. G. Lukina, S. Pelletier, P. Hubert, and E. Dellacherie, J. Colloid Interface Sci., 225, 505 (2000).

30. M. C. Miralles-Houzelle, P. Hubert, and E. Dellacherie, Langmuir, 17, 1384 (2001). 